\title{
Aging and the decline in health
}

\author{
Robin Holliday \\ Australian Academy of Science, Canberra, Australia; RandL.Holliday@bigpond.com
}

Received 4 November 2009; revised 14 December 2009; accepted 17 December 2009.

\begin{abstract}
The biological reasons for aging are now understood. Aging is the result of multiple stochastic events in molecules, cells, tissues and organs. These together produce the aged phenotype, senescence and ultimately death. Many of these changes can be directly linked to specific age-associated disease. However, there are also age-related changes that are not pathological. It can be said that aging has multiple causes, or is instead due to a general loss of molecular fidelity, that is, an increase in disorder. The complexity of organism means that they develop as ordered structures by obtaining energy from the environment. These ordered structures must be maintained by a wide variety of mechanisms which also depend on energy resources. Eventually these mechanisms fail, and senescence sets in. It is known that the efficiency of maintenance is correlated directly with the lifespan of different mammalian species. Also, these lifespans are inversely correlated with fecundity or reproductive potential. There is a trade off between investment of resources in maintenance of the body, or soma, and investment in reproduction.
\end{abstract}

Keywords: Aging; Senescence; Disease; Pathologies; Evolution

\section{INTRODUCTION}

It is now evident that aging is no longer an unsolved biological problem [1-6]. However, the relationship between natural aging in humans to age-associated diseases is controversial. Most books and reviews about aging completely ignore the vast literature on human age related pathologies. Also, most research on each of these pathologists is done by specialists who have no particular knowledge or interest in the process of ageing per se. An exception that relates late onset disease to aging is the excellent monograph The Oxford Textbook of Geriatric Medicine [7]. Hayflick [8] has argued that aging is an intrinsic process occurring in almost all animals, and that it is not directly related to particular age-associated pathologies. Instead, he states that aging makes an animal susceptible to these pathological events. In contrast, it is argued here and elsewhere $[2,5,9]$ that the process or processes of aging are responsible for most of these pathological changes. This leads to the loss or decline of health that is eventually lethal. Many diseases are the result of multiple molecular or cellular events. These events may occur over a long period of time, and it is their multiplicities that eventually produce the symptoms of disease. In other words many diseases can be due to time dependent multiple "hits" which are stochastic random events. There is an intermediate position which states that "senescence gives rise to disease, but disease does not give rise to senescence" [10], and also that the distinction between senescence and disease is blurred.

At the outset it is important to define some key words. Health is the state of being free from illness or injury. Aging is the process of growing old. Senescence is the condition or process of deterioration with age. Aging (ageing) and senescence are often used interchangeably. Disease is a disorder of structure or function which is not simply the result of specific injury. Pathology is the science of the causes and effects of diseases. (It is also the branch of medicine that deals with the laboratory examination of samples of body tissue for diagnostic or forensic purposes, but this is not relevant to the discussion here).

\section{THE BIOLOGICAL REASONS FOR AGING}

To understand aging one must first explain its biological origin and function. Organisms develop from the fertilized egg to become adults that are capable of reproduction. In the natural environment in which evolution occurred, animals are confronted with various hazards, for example, predators, insufficient food and water, or parasites and pathogens. Mortality is therefore high, so that 
most offspring are born to young adults rather than old ones. In this situation there is little, if any, natural selection for a long lifespan. Instead, Darwinian fitness is increased if resources are channeled into reproduction rather than preserving the body indefinitely [11-13]. It has been shown that in 47 mammalian species there is an inverse relationship between fertility and fecundity and longevity in a non-hazardous environment such as a zoo or under domestication [2,14]. (In the case of humans, longevity is highest in developed countries with good health care). It has also become evident that longevity is directly related to the maintenance of function of the various tissues and organs of the body.

There are many maintenance mechanisms, which will be listed but not reviewed here: 1) The repair of damage in DNA; 2) The degradation of abnormal protein molecules; 3) The defences against reactive oxygen species (ROS); 4) The immune system which provides defences against pathogens and parasites; 5) The detoxification of harmful chemicals in the diet; 6) Proofreading in the synthesis of macromolecules, which removes errors; 7) Wound healing, including the clotting of blood and the repair of broken bones; 8) Epigenetic controls for normal cell functions, and which also prevent the development of cancer; 9) Apoptosis, which removes potentially harmful cells; 10) Physiological homeostasis co-ordinating the functions of different cells, tissues and organs; 11) The grooming of hair and skin to remove harmful pests and parasites; 12) The storage of fat as an energy reserve.

In this list there is no reference to a central component of cell function, namely, RNA (apart from proofreading in its synthesis). This is largely due to lack of information. RNA transmits information in DNA to proteins, The translation of RNA into proteins depends on transfer RNAs and ribosomes (which consist of proteins and RNA). There is also the accurate splicing of RNA transcripts. The regulatory role of small RNAs has recently been demonstrated in normal cells. It would be surprising if 1) there were not important controls of all these functions, and 2) there were no age-related changes in RNA metabolism and function. However, they remain to be discovered.

Most of the listed maintenance mechanisms are scientific disciplines in their own right, and together they depend on a considerable proportion of the resources available to an animal. It should also be noted that a large number of genes are necessary to code for all the proteins and enzymes that are needed for each mechanism. These genes in one way or another have an effect on the process of aging.

There have been many comparative studies that clearly demonstrate that long-lived species have more efficient maintenance mechanisms than short lived ones. These have been comprehensively reviewed elsewhere [2], and since that time more evidence has been published [5,15-17]. Only a few examples can be mentioned here. The same chemical cross links occur more rapidly in bovine skin than human skin [18]. In rats, carcinomas are far more frequently than they do in humans, with an approximately 30-fold difference in the rate of onset [19]. Also, somatic mutations in lymphocytes increase about 10 fold during the lifespan of mice and humans. However, this increase occurs over about three years in mice and 80 years in humans [20]. It has been shown that the defences against ROS correlate with mammalian lifespan $[16,21,22]$. It has also been shown that these defences are much more efficient in the pigeon, which is long lived, than the rat, a short lived animal of similar size and metabolic rate [23]. A similar difference was demonstrated between small long-lived birds (canary and parakeet) and the short lived mouse [24]. From all these studies it can be concluded that it is the eventual decline in maintenance that brings about aging.

\section{CAUSES OF AGING}

How does the decline or loss of maintenance give rise to aging? To answer this question we need to consider many of the events that actually occur during aging $(2,15)$. It is known that chromosomal changes and also mutations increase during aging. There may be additional damage to DNA which is not recognized by repair enzymes and simply accumulates with time [25]. Many studies document deletions in mitochondrial DNA. Abnormalities in nuclear DNA can result in age-associated carcinomas. Altered proteins appear in many locations. Collagen and elastin become cross-linked, which is a cause of hardening of the arteries. The loss of elasticity of artery walls can increase blood pressure, and this can result in kidney damage and also strokes. Many types of protein react with glucose and other carbohydrates to produce advanced glycation products (AGEs). These are high molecular weight aggregates that can occur in many locations. There is also the accumulation of lipofuscin, also known as the "age pigment", which is a complex mixture of many degradation products. This is also seen in many tissues during aging. Recently there has been much interest in epigenetic events during aging, and particularly "epigenetic drift" [26-28]. These events may be due to changes in DNA methylation and histone modification, which in turn can change gene expression. There may be irregularities in hormone function or metabolism, for example, late onset diabetes is caused by a failure of the normal controls of insulin levels, or to changes in insulin receptors. In the brain, neurons may 
be lost or become abnormal, producing the amyloid plaques and neurofibrillary tangles which give rise to Alzheimer's disease and other dementias. In the vascular system, atheromatous plaques can form on the inner wall of the major arteries and these impair normal blood flow. This is a major cause of heart disease. The valves of the heart can become calcified, which is another component of the disease. Although muscle tissue can to some extent repair itself, over a long period of time cells are lost and not replaced. Aging is frequently associated with loss of hearing and sight. The long lived protein crystallin becomes denatured and loses transparency, and this result in the appearance of lens cataracts. Retinopathy is largely due to the failure to remove protein aggregates that are the end products of the continual turnover of photoreceptors in the cells of the retina. The age-associated diseases of osteoporosis and osteoarthritis, are due to a decline in normal bone metabolism and the accumulation of damage in bone joints. Another example of multiple events giving rise to disease is the gradual loss of kidney glomeruli and eventually renal failure.

\section{MULTIPLE EVENTS}

The previous section is only a summary of some of the changes that can occur during aging. They are sufficient to demonstrate that multiple events at the molecular and cellular level can bring about very significant changes in tissues and organs. These in turn can bring about ill health and disease during ageing. However, not all age associated multiple events are pathological. A good example is the whitening of hair. This is due to the loss of melanocytes in hair follicles, and the loss of hair follicles themselves results in baldness. One of the most obvious effects of aging is on the skin, and this provides a rough measure of a person's age. Skin changes are due to loss of elasticity, wrinkling, and often the accumulation of pigmented "age spots". Leaving aside skin cancers, these cumulative effects are simply part of the aging phenotype, and do not impair health. A third example is the loss of muscle strength with age, which by itself is not a harmful change, but is can lead to other problems such as falls and broken bones, particularly if the individual also has osteoporosis.

It is interesting that these outward manifestations of aging are not pathological. It is the internal changes that eventually produce age-associated disease and a senescent phenotype. The longevity of identical twins is more similar that the longevities of sibs. Also, inbred mice which are genetically identical and live in the same environment have quite variable lifespans, and the survival curve of a population of these mice is an S-shaped curve. There is a plateau with no deaths, then slowly increasing mortality, followed by a steeper increase in mortality. Finally, the slope of the curve flattens out and a few "outliers" achieve the longest lifespan [29] If this curve is plotted on a logarithmic scale, there is again a plateau followed by an exponential decline in surviving animals. This is very similar to observations in radiology, where, for example, a population of viruses is irradiated. Again there is a plateau in survival, followed by an exponential decline in survival. This is an example of a multiple hit survival curve. The same can be said of many of the vulnerable components of the body, and taken together this constitutes aging of the whole body. Obviously, there is not complete synchrony in the change and loss of function of one or another tissue or organ system.

This can gives rise to a common misconception. For example, it has frequently been said that dementia has nothing to do with ageing because many old people retain their mental facilities throughout their lifespan. The same can be said of carcinomas, cardiovascular disease, and so on. A further related and interesting point is that death certificates must specify a particular cause or causes of death, it is not permissible for a physician to use the term "natural aging".

During long periods of evolution some animal species reduce their lifespan, whereas others increase it. Since aging is multi-causal, the evolved changes in the efficiency of maintenance must depend on a degree of synchrony in the rate that tissues and organs gradually become senescent. There would be no selective advantage if one organ system increased (or reduced) its survival time, whilst others did not. This was first clearly stated by John Maynard Smith nearly fifty years ago [30].

\section{THE LOSS OF MOLECULAR FIDELITY}

In his book What is Life? Schrodinger [31] discussed the complexity of living organisms. The second law of thermodynamics states that ordered states of atoms and molecules will eventually become disordered. Schrodinger wrote about organisms feeding on negative entropy. This means that they depend on energy to maintain their complex structures, which is a contravention of the second law. It is obvious that this is not a permanent situation because aging eventually results in death, and it is after this that disorder prevails. Instead of stating that there are multiple causes of aging, one can instead regard the whole process of aging to have just one cause, and this is the loss of molecular fidelity, and gain in entropy. This is the viewpoint of Hayflick $[4,8]$.

There is, however, no radical distinction between this one cause of aging and the multiple causes that have been discussed here. The analogy of a motor car helps to explain this. The car is a complex machine that requires 
continual service and maintenance. With time it is subject to wear and tear in its component parts which are to some extent independent of each other, but are also essential for its normal function.

We can equate the loss of molecular fidelity or increasing molecular disorder with wear and tear. The component parts of a car cannot be expected to last indefinitely, for example, the gearbox, the electrical system, the cooling system, the engine, and so on. With time the defects increase and are more and more expensive to repair. At a given point in time the car is not worth repairing and reaches the end of its working life. We can conclude that the multiple parts of a car deteriorate at a given rate, and its life ends as a result on innumerable cumulative defects, which are the equivalent of "molecular infidelity" in an organism. It is also significant to note that a vintage car can be maintained indefinitely, but at huge expense. Similarly an animal body could, in principle, be maintained indefinitely, but a cost in resources that would be selectively disadvantageous. Thus all animals, except a few of the simplest, have finite lifespan.

\section{CONCLUSIONS}

The biological reasons for aging are no longer a mystery. The adult organism is a structure capable of reproduction for a given period, but in a natural environment, most offspring are born to young adults because environmental hazards limit the lifespan of parents. The energy resources available to every animal are used for general metabolism, for reproduction and also to maintain the body, or soma. Normal metabolism is obviously essential for life, but there is a trade off between the investment of resources in reproduction and in body maintenance, which varies between species. Thus, short lived small species, such as rodents, can have many offspring, whilst large long lived species have few offspring.

In the case of the human species, a great deal is known about the changes that occur with aging. Many of these changes affect vital functions which result in a decline in health and eventually to events that cannot be treated at all. Nevertheless, the last few months of life very often depend on medical resources that are increasingly expensive, especially in developed countries. In the end, all care and treatments fail, and the elderly individual dies. It is true to say that aging is almost always associated with a decline in health leading to death.

\section{REFERENCES}

[1] Hayflick, L. (1996) How and Why We Age. Ballantine Books, New York.
[2] Holliday, R. (1995) Understanding Ageing. Cambridge University Press, Cambridge.

[3] Austad, S.N. (1997) Why We Age. John Wiley, New York.

[4] Hayflick, L. (2007) Biological aging is no longer an unsolved problem. Annals of the New York Academy of Sciences, 1100(1), 1-13.

[5] Holliday, R. (2006) Aging is no longer an unsolved problem in biology. Annals of the New York Academy of Sciences, 1067(1), 1-9.

[6] Kirkwood, T.B. (2005) Understanding the odd science of aging. Cell, 120(4), 437-447.

[7] Evans, G.J., Franklin W.T., Beattie, B., Michel, J.P. and Wilcock, G.K. (2000) The Oxford Textbook of Geriatric Medicine. Oxford University Press, Oxford.

[8] Hayflick, L., (2004) The not-so-close relationship between biological aging and age-associated pathologies in humans. Journal of Gerontology: Biological Sciences, 59 (6), 547-550.

[9] Holliday, R. (2004) The close relationship between biological aging and age-associated pathologies in humans. Journal of Gerontology: Biological Sciences, 59(6), 543546.

[10] Carnes, B.A., Staats, D.O. and Sonntag, W.G. (2009) Does senescence give rise to disease? Mechanisms of Ageing and Development, 129(12), 693-699.

[11] Kirkwood, T.B.L. (1977) The evolution of ageing. Nature, 270(5635), 301-304.

[12] Kirkwood, T.B. and Holliday, R. (1979) The evolution of ageing and longevity. Proceedings of the Royal Society London B: Biological Sciences, London, 205(1161), 532546.

[13] Kirkwood, T.B.L. and Holliday, R. (1986) Ageing as a consequence of natural selection. In. Collins, K.J. and Bittles, A.H., Eds., Biology of Human Ageing, Cambridge University Press, Cambridge.

[14] Holliday, R. (1994) Longevity and fecundity in eutherian mammals. In. Rose, M.R. and Finch, C.E. Eds., Genetics and the Evolution of Aging, Kluwer Academic, Dordrecht.

[15] Holliday, R., (2004) The multiple and irreversible causes of aging. Journal of Gerontology: Biological Sciences, 59(6), 568-572.

[16] Barja, G., (2004) Aging in vertebrates, and the effect of calorie restriction: a mitochondrial free radical production-DNA damage mechanism. Biological Reviews of the Cambridge Philosophical Society, 79(2), 235-251.

[17] Kapahi, B., Boulton, M.E. and Kirkwood, T.B.L. (1999) Positive correlation between mammalian lifespan and cellular resistance to stress. Free Radical Biology and Medicine, 26(5-6), 495-500.

[18] Yamauchi, M., Woodley, D.T. and Mechanic, G.L. (1988) Aging and cross-linking of skin collagen. Biochemical and Biophysical Research Communications, 152(2), 898903.

[19] Ames, B.N., Saul, R.L., Schwiers, E., Adelman, R. and Cathcart, R. (1985) Oxidative damage as related to cancer and aging: the assay of thymine glycol, thymidine glycol and hydroxymethyl uracil in human and rat urine. In. Sohal, R.S., Birnbaum, L.S. and Cutler, R.G.., Eds., Molecular Biology of Aging, Raven Press, New York.

[20] Morley, A.A., (1998) Somatic mutations and aging. Annals of the New York Academy of Sciences, 854, 20-22. 
[21] Pamplona, R., Portero-Ortin, M., Ruiz, C., Gredilla, R., Herrero, A. and Barja, G. (2000) Double bond content of phospholipids and lipid peroxidation negatively correlate with maximum longevity in the heart of mammals. Mechanisms of Ageing and Development, 112(3), 169183.

[22] Barja, G. and Herrero, A. (2000) Oxidative damage to mitochondria is inversely correlated to maximum lifespan in the heart and brain of mammals. Journal of the Federation of American Societies for Experimental Biology, 14(2), 312-318.

[23] Pamplona, R., Portero-Otin, M., Requena, J.R., Thorpe, S.R., Herrero, A. and Barja, G. (1999) A low degree of fatty acid unsaturation leads to lower lipid peroxidation and lipoxidation derived proteiun modification in heart mitochondria in the longevous pigeon than the short lived rat. Mechanisms of Ageing and Development, 106 (3), 283-296.

[24] Pamplona, R., Portero-Otin, M., Riba, D., Gredilla, R., Herrero, A. and Barja, G. (1999) Heart fatty acid unsaturation and lipid peroxidation, and aging rate, are lower in the parakeet and canary than in the mouse. Aging Clinical and Experimental Research, 11, 44-49.

[25] Lindahl, T. (1993) Instability and decay of the primary structure of DNA. Nature, 362(6422), 709-715

[26] Fraga, M.F., Ballistar, E., Paz, M.F., et al. (2005) Epigenetic differences arise during the lifetime of monozygotic twins. Proc. Nat. Acad. Sci. USA, 102(30), 10604-10609.

[27] Martin, G.M. (2005) Epigenetic drift in aging identical twins. Proceedings of the National Academy Science USA, 102, 10413-10414.

[28] Tollefsbol, T. (2009) Epigenetics of aging. Springer Science, Humana Press, New Jersey.

[29] Zurcher, C., Van Zwieten, M.J., Solleveld, H.A. and Hollander, C.F. (1982) Ageing research. In. Foster H. L., Small, J.D. and Fox, J.G. Eds., The Mouse in Biomedical Research, Academic Press, New York, 4, 11-35.

[30] Smith, M.J. (1962) The causes of ageing. Proceedings of the Royal Society London B: Biological Sciences, London, 157(966), 115-127.

[31] Schrodinger, E. (1944) What is life? Cambridge University Press. Cambridge. 